\title{
Dose Response Study
}

National Cancer Institute

\section{Source}

National Cancer Institute. Dose Response Study. NCI Thesaurus. Code C127803.

A study of the effect of dose changes on the efficacy of a drug in order to determine the dose-response relationship and optimal dose of a therapy. 